\title{
Left ventricular response after cardiac resynchronization therapy is related to early left atrial volume reduction
}

In-Jeong $\mathrm{Cho}^{1}$, Jae-Sun $\mathrm{Uhm}^{2}$, Jaewon $\mathrm{Oh}^{2}$, Jong-Ho Nam², Hee Tae $\mathrm{Yu}^{2}$, Taehoon $\mathrm{Kim}^{2}$, Boyoung Joung ${ }^{2}$, and Seok-Min Kang ${ }^{2}$

${ }^{1}$ Division of Cardiology, Department of Internal Medicine, Ewha Womans University Seoul Hospital, Seoul; ${ }^{2}$ Division of Cardiology, Department of Internal Medicine, Severance Cardiovascular Hospital, Yonsei University College of Medicine, Seoul, Korea

Received: November 29, 2018 Revised : March 30, 2019

Accepted: April 3, 2019

\section{Correspondence to}

Seok-Min Kang, M.D.

Division of Cardiology, Severance Cardiovascular Hospital, Yonsei University College of Medicine, 50 Yonsei-ro, Seodaemun-gu,

Seoul 03722, Korea

Tel: +82-2-2228-8450

Fax: +82-2-2227-7722

E-mail: smkang@yuhs.ac
Background/Aims: The current study aimed to elucidate a time-course change in left atrial volume after cardiac resynchronization therapy (CRT) and to verify factors associated with left atrial volume reduction (LAVR) and its prognostic implications.

Methods: The records of 97 patients were retrospectively reviewed after CRT. Echocardiographic data were analyzed at baseline before CRT, at early follow-up $(\mathrm{FU})(\leq 1$ year, median 6 months), and at late FU (median 30 months). Left ventricular volume response (LVVR) was defined as $15 \%$ reduction in left ventricular (LV) end-systolic volume (ESV). LAVR was classified into two groups by the median value at early FU: LAVR ( $\geq 7.5 \%)$ and no LAVR (< $7.5 \%)$.

Results: LV ESV index continuously decreased from baseline to early FU and from early FU to late FU $\left(106.1 \pm 47.4 \mathrm{~mL} / \mathrm{m}^{2}\right.$ vs. $87.6 \pm 51.6 \mathrm{~mL} / \mathrm{m}^{2}$ vs. $\left.72.5 \pm 57.1 \mathrm{~mL} / \mathrm{m}^{2}\right)$. LA volume index decreased from baseline to early FU, but there were no reductions thereafter $\left(51.8 \pm 21.9 \mathrm{~mL} / \mathrm{m}^{2}\right.$ vs. $45.1 \pm 19.6 \mathrm{~mL} / \mathrm{m}^{2}$ vs. $\left.44.9 \pm 23.0 \mathrm{~mL} / \mathrm{m}^{2}\right)$. The only echocardiographic factor associated with LAVR was change in E velocity (odds ratio $[\mathrm{OR}], 1.04 ; p=0.002)$. Early LAVR (OR, 10.05; $p=0.002$ ) was an independent predictor for late LVVR.

Conclusions: LAVR was related to reduction in E velocity, suggesting its relation with optimization of LV filling pressure. Early LAVR was a predictor for LVVR to CRT in long-term FU.

Keywords: Atrial remodeling; Ventricular remodeling; Cardiac resynchronization therapy

\section{INTRODUCTION}

Cardiac resynchronization therapy (CRT) improves symptoms and survival in patients with medically refractory heart failure and left ventricular (LV) systolic dysfunction with electrical dyssynchrony [1]. The beneficial clinical outcomes have mainly been attributed to significant reduction in LV end-systolic volume (ESV) [2]. Several stud- ies have indicated that reduced left atrial (LA) size after CRT device implantation was also associated with favorable clinical outcomes [3,4], suggesting that reductions in both LA and LV volumes predict clinical outcomes in patients with an implanted CRT device. However, individual variability of reductions in LA and LV volumes after CRT has been described and a considerable proportion of patients showed discordant reductions in LA 
and LV volumes in a previous study [5].

CRT restores the homogeneous LV contraction pattern, thus reducing LV ESV and improving systolic function. CRT induces LV volume reduction (LVVR) that can be sustained for up to 3 years [6]. However, the time-course of changes in LA volume has not been well investigated after CRT. LA volume is a morphologic expression of the severity and chronicity of LV diastolic dysfunction and LV filling pressure because the left atrium is directly exposed to pressures in the LV that increase with decreased LV compliance during diastole [7]. Therefore, hemodynamic factors during diastole may determine LA volume reduction (LAVR) after CRT and the time-course of LAVR may be different from that of LVVR.

The objectives of this study were (1) to assess changes in LAVR after CRT device implantation, (2) to investigate the time-course relationship between LAVR and LVVR, and (3) to identify echocardiographic factors associated with LAVR and their clinical implications.

\section{METHODS}

\section{Study population}

Medical records of 97 consecutive patients who underwent CRT-pacemaker or CRT-defibrillator implantation between February 2010 and October 2016 at an adult heart failure clinic of a tertiary referral hospital (Severance Cardiovascular Hospital, Seoul, Korea) were retrospectively reviewed. Echocardiographic data at baseline before CRT device implantation, at early follow-up (FU) ( $\leq 1$ year after device implantation), and at late FU (last echocardiography acquired during FU period) were analyzed. Patients were classified into two groups as follows according to the median value of LAVR at early FU: (1) LAVR and (2) No LAVR. LV response to CRT was defined as an absolute reduction in LV ESV $\geq 15 \%$ at FU echocardiography [5]. This study was approved by the Institutional Review Board of Yonsei University, Severance Hospital, Seoul, Korea (IRB No: 1-2013-0061). Written informed consent by the patients was waived due to a retrospective nature of our study.

\section{Echocardiographic methods}

LV end-diastolic volume (EDV) and ESV were measured using biplane disc methods from apical four-chamber and two-chamber views [8]. LV end-diastolic volume index (EDVI) and end-systolic volume index (ESVI) were LV EDV and ESV indexed for body surface area (BSA). LV ejection fraction (EF) was calculated using LV volumes. LA volume was calculated using the biplane area method. LA volume index (LAVI) was LA volume indexed BSA. Mitral inflow velocities were obtained using pulse wave Doppler in the apical four-chamber view. Early mitral inflow velocity (E) and late diastolic velocity were also measured, and the early mitral annular $\left(e^{\prime}\right)$ velocity was recorded from the septal mitral annulus. The $\mathrm{E} / \mathrm{e}^{\prime}$ ratio was then calculated. Diastolic function was assessed by transmitral and tissue Doppler. According to mitral inflow and tissue Doppler pattern, patients were divided into grade 1 to 3 as follows: grade 1, relaxation abnormality; grade 2, pseudonormalization; and grade 3, restrictive LV filling pattern [9]. The calculated systolic pulmonary artery pressure was defined as: $4 \times$ (maximum velocity of tricuspid regurgitant jet $)^{2}+$ right atrial pressure. Right atrial pressure was estimated by measuring the inferior vena cava diameter and its response to inspiration [10].

Mitral regurgitation (MR) was graded as grade o to 4 . The qualitative, semiquantative, and quantitative parameters used to grade MR were standard criteria based on the American Society of Echocardiography recommendations [11]. Changes between baseline and early FU $(\Delta)$ values were calculated for LV ESVI ( $\Delta$ LV ESVI), LV EF $(\Delta \mathrm{LV} \mathrm{EF}), \mathrm{LAVI}(\Delta \mathrm{LAVI}), \mathrm{E}(\Delta \mathrm{E})$ velocity, $\mathrm{E} / \mathrm{e}^{\prime}\left(\Delta \mathrm{E} / \mathrm{e}^{\prime}\right)$, and $\operatorname{MR}(\Delta \mathrm{MR})$ grade.

\section{Clinical events}

Patients who underwent baseline echocardiography were followed up across a median of 22 months (interquartile range, 12 to 33) for a composite endpoint that included cardiac death and inpatient admission for heart failure. The occurrence of clinical events was ascertained by reviewing hospital records and by telephone interview, as necessary.

\section{Statistical analysis}

Variables are presented as mean \pm standard deviation or number (\%). Paired $t$ tests were used to compare time-course changes for echocardiographic variables after CRT device implantation. Groups were compared 
using chi-square statistics for categorical variables and Student's $t$ test for continuous variables. If distributions were skewed, the Mann-Whitney U or Kruskal-Wallis nonparametric tests were used as appropriate. The correlation among changes in echocardiographic variables was assessed using Pearson's correlation coefficient for

Table 1. Baseline characteristics of study population

\begin{tabular}{|c|c|c|c|c|}
\hline \multirow{2}{*}{ Variable } & \multirow{2}{*}{ Total population $(\mathrm{n}=97)$} & \multicolumn{3}{|c|}{ LAVR at early FU } \\
\hline & & $\operatorname{LAVR}(n=48)$ & No LAVR $(n=49)$ & $p$ value \\
\hline Age, yr & $66 \pm 12$ & $66 \pm 11$ & $66 \pm 13$ & 0.947 \\
\hline Women & $46(47.4)$ & $22(42.5)$ & $24(49.0)$ & 0.756 \\
\hline Body surface area, $\mathrm{m}^{2}$ & $1.63 \pm 0.19$ & $1.62 \pm 0.17$ & $1.63 \pm 0.21$ & 0.927 \\
\hline Ischemic & $12(12.4)$ & $4(8.3)$ & $8(16.8)$ & 0.232 \\
\hline CRT-P & $24(24.7)$ & $15(31.3)$ & $9(18.4)$ & 0.142 \\
\hline \multicolumn{5}{|l|}{ Baseline ECG findings } \\
\hline LBBB & $69(71.1)$ & $34(70.8)$ & $35(71.4)$ & 0.948 \\
\hline QRS duration, msec & $163.6 \pm 21.9$ & $166.3 \pm 22.4$ & $160.8 \pm 21.2$ & 0.216 \\
\hline \multicolumn{5}{|l|}{ Medication } \\
\hline ACEI/ARB & $86(88.7)$ & $40(83 \cdot 3)$ & $46(93 \cdot 3)$ & 0.102 \\
\hline Beta-blocker & $65(67.0)$ & $36(75.0)$ & $29(59.2)$ & 0.098 \\
\hline Spironolactone & $58(59.8)$ & $30(62.5)$ & $28(57.1)$ & 0.590 \\
\hline \multicolumn{5}{|l|}{ Echocardiography } \\
\hline LV EDVI, mL/m² & $138.2 \pm 53.5$ & $140.2 \pm 49.7$ & $136.2 \pm 57.5$ & 0.716 \\
\hline LV ESVI, mL/m² & $106.1 \pm 47.4$ & $108.6 \pm 45 \cdot 7$ & $103.7 \pm 49 \cdot 3$ & 0.616 \\
\hline LV EF, \% & $24.6 \pm 6.2$ & $24.2 \pm 7.1$ & $24.9 \pm 5.2$ & 0.578 \\
\hline LAVI, $\mathrm{mL} / \mathrm{m}^{2}$ & $51.8 \pm 21.9$ & $55 \cdot 4 \pm 23 \cdot 3$ & $48.3 \pm 20.1$ & 0.110 \\
\hline E velocity, $\mathrm{cm} / \mathrm{sec}$ & $77.2 \pm 29.5$ & $78.6 \pm 33.0$ & $75.9 \pm 25.8$ & 0.657 \\
\hline $\mathrm{e}^{\prime}$ velocity, $\mathrm{cm} / \mathrm{sec}$ & $4.1 \pm 1.8$ & $4.1 \pm 1.8$ & $4.0 \pm 1.7$ & 0.692 \\
\hline $\mathrm{E} / \mathrm{e}^{\prime}$ & $21.1 \pm 10.7$ & $21.5 \pm 12.2$ & $20.7 \pm 9.1$ & 0.712 \\
\hline SPAP, mmHg & $38.2 \pm 16.2$ & $41.7 \pm 18.7$ & $34.4 \pm 14.6$ & 0.059 \\
\hline MR grade & & & & 0.682 \\
\hline Grade o & $27(27.8)$ & $14(29.2)$ & $13(26.5)$ & \\
\hline Grade 1 & $22(22.7)$ & $8(16.7)$ & $14(28.6)$ & \\
\hline Grade 2 & $21(21.6)$ & $12(25.0)$ & $9(18.4)$ & \\
\hline Grade 3 & $22(22.7)$ & $11(22.9)$ & $11(22.4)$ & \\
\hline Grade 4 & $5(5.2)$ & $3(6.2)$ & $2(4.1)$ & \\
\hline Diastolic function & & & & 0.589 \\
\hline Grade 1 & $47(48.5)$ & $23(47.9)$ & $24(49.0)$ & \\
\hline Grade 2 & $11(11.3)$ & $4(8 \cdot 3)$ & $7(14 \cdot 3)$ & \\
\hline Grade 3 & $39(40.2)$ & $21(43.8)$ & $18(36.7)$ & \\
\hline
\end{tabular}

Values are presented as mean $\pm \mathrm{SD}$ or number (\%).

LAVR, left atrial volume reduction; FU, follow-up; CRT-P, cardiac resynchronization therapy-pacemaker; ECG, electrocardiography; LBBB, left bundle branch block; ACEI, angiotensin-converting enzyme inhibitor; ARB, angiotensin receptor blocker; LV, left ventricular; EDVI, end-diastolic volume index; ESVI, end-systolic volume index; EF, ejection fraction; LAVI, left atrial volume index; E velocity, early mitral inflow velocity; e' velocity, early mitral annular velocity; SPAP, systolic pulmonary artery pressure; MR, mitral regurgitation. 

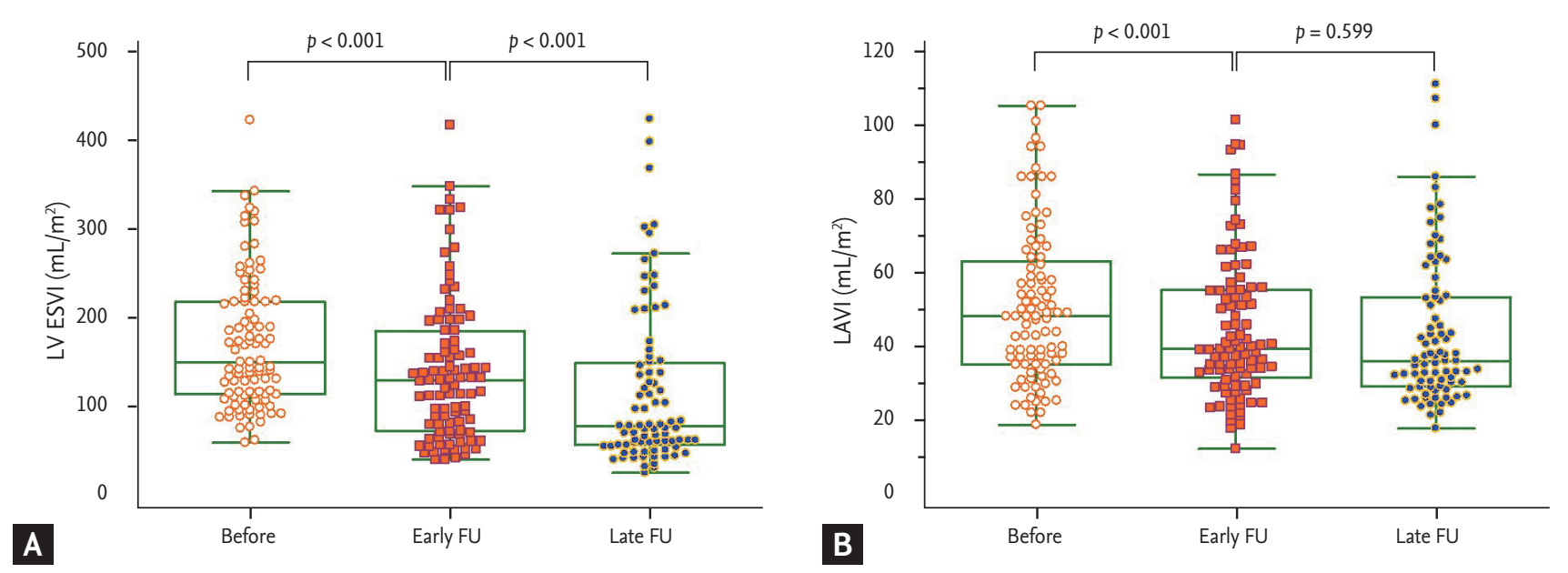

Figure 1. Time course changes in left ventricle and left atrium. (A) Left ventricular end-diastolic volume index (LV ESVI). (B) Left atrial volume index (LAVI). FU, follow-up.

variables with a normal distribution and Spearman's correlation coefficient for those with a non-normal distribution. To determine the independent factors associated with LAVR, univariate and multivariate logistic regression analyses were performed. A $p$ value $<0.05$ was considered statistically significant.

\section{RESULTS}

Echocardiography was performed in all 97 patients at baseline and at early FU (100.0\%) and in 83 patients at late FU (85.6\%). Late FU echocardiography was lost in 14 patients, including seven patients with cardiovascular mortality within 1 year after CRT and seven patients who had not undergone late FU echocardiography after 1 year. Early and late FU echocardiography were performed at a median of 6 months (interquartile range, 4 to 7) and 30 months (interquartile range, 21 to 38 ), respectively.

\section{Baseline characteristics}

Table 1 shows baseline characteristics of the study population. The mean age was $66 \pm 12$ years, and 46 patients (47.4\%) were women. Ischemic heart failure was present in 12 patients (12.4\%). Left bundle branch block (LBBB) was identified in 69 patients (71.1\%), and mean QRS duration was $163.6 \pm 21.9 \mathrm{msec}$. CRT pacemakers were implanted in 24 patients (24.7\%). Median LAVR at early FU was $7.5 \%$. There were no significant differences in baseline characteristics including diverse echocardiographic variables between LAVR and no LAVR groups.

\section{Echocardiographic data}

Mean LV ESVI and LAVI at baseline were 106.1 \pm 47.4 and $51.8 \pm 21.9 \mathrm{~mL} / \mathrm{m}^{2}$, respectively. As shown in Fig. 1 , LV ESVI continuously decreased from baseline to early $\mathrm{FU}\left(106.1 \pm 47.4 \mathrm{~mL} / \mathrm{m}^{2}\right.$ vs. $\left.87.6 \pm 51.6 \mathrm{~mL} / \mathrm{m}^{2}, p<0.001\right)$ and from early to late FU $\left(87.6 \pm 51.6 \mathrm{~mL} / \mathrm{m}^{2}\right.$ vs. $72.5 \pm$ $\left.57.1 \mathrm{~mL} / \mathrm{m}^{2}, p<0.001\right)$. In contrast, LAVI significantly decreased from baseline to early FU $\left(51.8 \pm 21.9 \mathrm{~mL} / \mathrm{m}^{2}\right.$ vs. $\left.45.1 \pm 19.6 \mathrm{~mL} / \mathrm{m}^{2}, p<0.001\right)$, but there were no significant reductions thereafter $\left(45.1 \pm 19.6 \mathrm{~mL} / \mathrm{m}^{2}\right.$ vs. $44.9 \pm$ $23.0 \mathrm{~mL} / \mathrm{m}^{2}, p=0.599$ ).

Echocardiographic data at baseline, early FU, and late FU are shown in Table 2. LV EDVI, LV EF, MR grade, and diastolic function grade showed continuous changes from baseline to early FU and from early to late FU, similar to the changing pattern of LV ESVI. E velocity and $\mathrm{E} / \mathrm{e}^{\prime}$ decreased from baseline to early $\mathrm{FU}$, but there were no reductions thereafter, similar to the changing pattern of LAVI. There were no significant changes in $\mathrm{e}^{\prime}$ velocity from baseline to early $\mathrm{FU}(4.1 \pm 1.8 \mathrm{~cm} / \mathrm{sec}$ vs. 3.9 $\pm 1.3 \mathrm{~cm} / \mathrm{sec}, p=0.216)$, and $\mathrm{e}^{\prime}$ velocity increased at late FU $(3.9 \pm 1.3 \mathrm{~cm} / \mathrm{sec}$ vs. $4.3 \pm 1.3 \mathrm{~cm} / \mathrm{sec}, p=0.025)$.

\section{Factors associated with LAVR at early FU}

Fig. 2 demonstrates simple correlations of $\triangle \mathrm{LAVI}$ to 
Table 2. Comparison of time-course changes in echocardiographic variables

\begin{tabular}{|c|c|c|c|}
\hline Variable & Baseline $(\mathrm{n}=97)$ & Early FU (n = 97) & Late $\mathrm{FU}(\mathrm{n}=83)$ \\
\hline LV EDVI, $\mathrm{mL} / \mathrm{m}^{2}$ & $138.2 \pm 53.5$ & $120.2 \pm 81.1^{a}$ & $108.1 \pm 62.3^{\mathrm{a}, \mathrm{b}}$ \\
\hline LV ESVI, mL/m² & $106.1 \pm 47.4$ & $87.6 \pm 51.6^{a}$ & $72.5 \pm 57.1^{\mathrm{a}, \mathrm{b}}$ \\
\hline LV EF, \% & $24.6 \pm 6.2$ & $31.1 \pm 10.9^{\mathrm{a}}$ & $38.1 \pm 13.8^{\mathrm{a}, \mathrm{b}}$ \\
\hline $\mathrm{LAVI}, \mathrm{mL} / \mathrm{m}^{2}$ & $51.8 \pm 21.9$ & $45.1 \pm 19.6^{a}$ & $44.9 \pm 23.0^{a}$ \\
\hline E velocity, $\mathrm{cm} / \mathrm{sec}$ & $77.2 \pm 29.5$ & $64.2 \pm 27.0^{a}$ & $64.9 \pm 23.5^{\mathrm{a}}$ \\
\hline $\mathrm{e}^{\prime}$ velocity, $\mathrm{cm} / \mathrm{sec}$ & $4.1 \pm 1.8$ & $3.9 \pm 1.3$ & $4 \cdot 3 \pm 1.3^{b}$ \\
\hline $\mathrm{E} / \mathrm{e}^{\prime}$ & $21.1 \pm 10.7$ & $18.1 \pm 9.8^{a}$ & $16.8 \pm 10.0^{\mathrm{a}}$ \\
\hline SPAP, mmHg & $38.2 \pm 16.2$ & $37.1 \pm 16.5$ & $33.0 \pm 11.2$ \\
\hline \multicolumn{4}{|l|}{ MR grade } \\
\hline Grade o & $27(27.8)$ & $40(41.2)^{\mathrm{a}}$ & $39(47 \cdot 0)^{a, b}$ \\
\hline Grade 1 & $22(22.7)$ & $20(20.6)$ & $23(27 \cdot 7)$ \\
\hline Grade 2 & $21(21.6)$ & $19(19.6)$ & $7(8.4)$ \\
\hline Grade 3 & $22(22.7)$ & $15(15 \cdot 5)$ & $11(13 \cdot 3)$ \\
\hline Grade 4 & $5(5.2)$ & $3(3.1)$ & $3(3.6)$ \\
\hline \multicolumn{4}{|l|}{ Diastolic function } \\
\hline Grade 1 & $47(48.5)$ & $68(70.1)^{\mathrm{a}}$ & $5^{8}(69 \cdot 9)^{\mathrm{a}, \mathrm{b}}$ \\
\hline Grade 2 & $11(11.3)$ & $7(7.2)$ & $12(14 \cdot 5)$ \\
\hline Grade 3 & $39(40.2)$ & $22(22.7)$ & $13(15.7)$ \\
\hline
\end{tabular}

Values are presented as mean \pm SD or number (\%).

FU, follow-up; LV, left ventricular; EDVI, end-diastolic volume index; ESVI, end-systolic volume index; EF, ejection fraction; LAVI, left atrial volume index; E velocity, early mitral inflow velocity; e' velocity, early mitral annular velocity; SPAP, systolic pulmonary artery pressure; MR, mitral regurgitation.

a $p<0.05$ compared with baseline.

$\mathrm{b} p<0.05$ compared with early follow-up.

$\Delta \mathrm{E}$ velocity, $\Delta \mathrm{E} / \mathrm{e}^{\prime}, \Delta \mathrm{LV}$ ESVI, and $\Delta \mathrm{LV} \mathrm{EF}$. There were significant positive correlations between $\triangle \mathrm{LAVI}$ and $\Delta$ E velocity $(r=0.616, p<0.001)$ and between $\Delta$ LAVI and $\Delta \mathrm{E} / \mathrm{e}^{\prime}(r=0.494, p<0.001)$. However, no significant correlations were found between $\triangle \mathrm{LAVI}, \Delta \mathrm{LV}$ ESVI, and $\triangle \mathrm{LV}$ EF. Table 3 shows the results of logistic regression analysis for factors associated with LAVR at early FU. In univariate analysis, $\Delta \mathrm{LV}$ ESVI, $\triangle \mathrm{EF}, \Delta \mathrm{E}$ velocity, and $\Delta \mathrm{E} / \mathrm{e}^{\prime}$ were associated with LAVR (all $p<0.05$ ), whereas baseline echocardiographic variables did not show any association with LAVR. In multivariate analysis, $\Delta \mathrm{E}$ velocity was the only independent factor associated with LAVR, even after adjusting for $\Delta \mathrm{LV}$ ESVI, $\Delta \mathrm{LV} \mathrm{EF}, \Delta \mathrm{E} / \mathrm{e}^{\prime}$, and $\Delta \mathrm{MR}$ grade (odds ratio [OR], 1.04; 95\% CI, 1.02 to $1.07 ; p=0.002)$.

Fig. 3 compares diastolic function before and early after CRT for the LAVR groups. There were no significant differences in diastolic function between the two groups at baseline before CRT $(p=0.589)$. There were more patients with grade $1(87.5 \%$ vs. $53.1 \%)$ than grade 3 (10.4\% vs. $35.4 \%$ ) diastolic function in the LAVR group $(p=0.001)$.

\section{Factors associated with LV response to CRT}

Among all 97 patients after CRT, 55 patients (56.7 \%) had early LV response. The number of LV responses increased at FU, and 60 of 83 patients (72.3 \%) had LV response at late FU. Fig. 4 compares the prevalence of LV response between LAVR groups according to FU period. There were no significant differences in LV response rates at early FU between the two groups (64.6\% vs. $49.0 \%, p=0.121)$. However, LV response rate at late FU was significantly higher in the LAVR group at early FU (83.3\% vs. 61.0\%, $p=0.029)$. $\Delta$ LV ESVI at late FU was higher in those with early LAVR compared to those without early LAVR $\left(-26.3 \pm 29.0 \mathrm{~mL} / \mathrm{m}^{2}\right.$ vs. $-11.0 \pm 24.8$ 


\section{A}
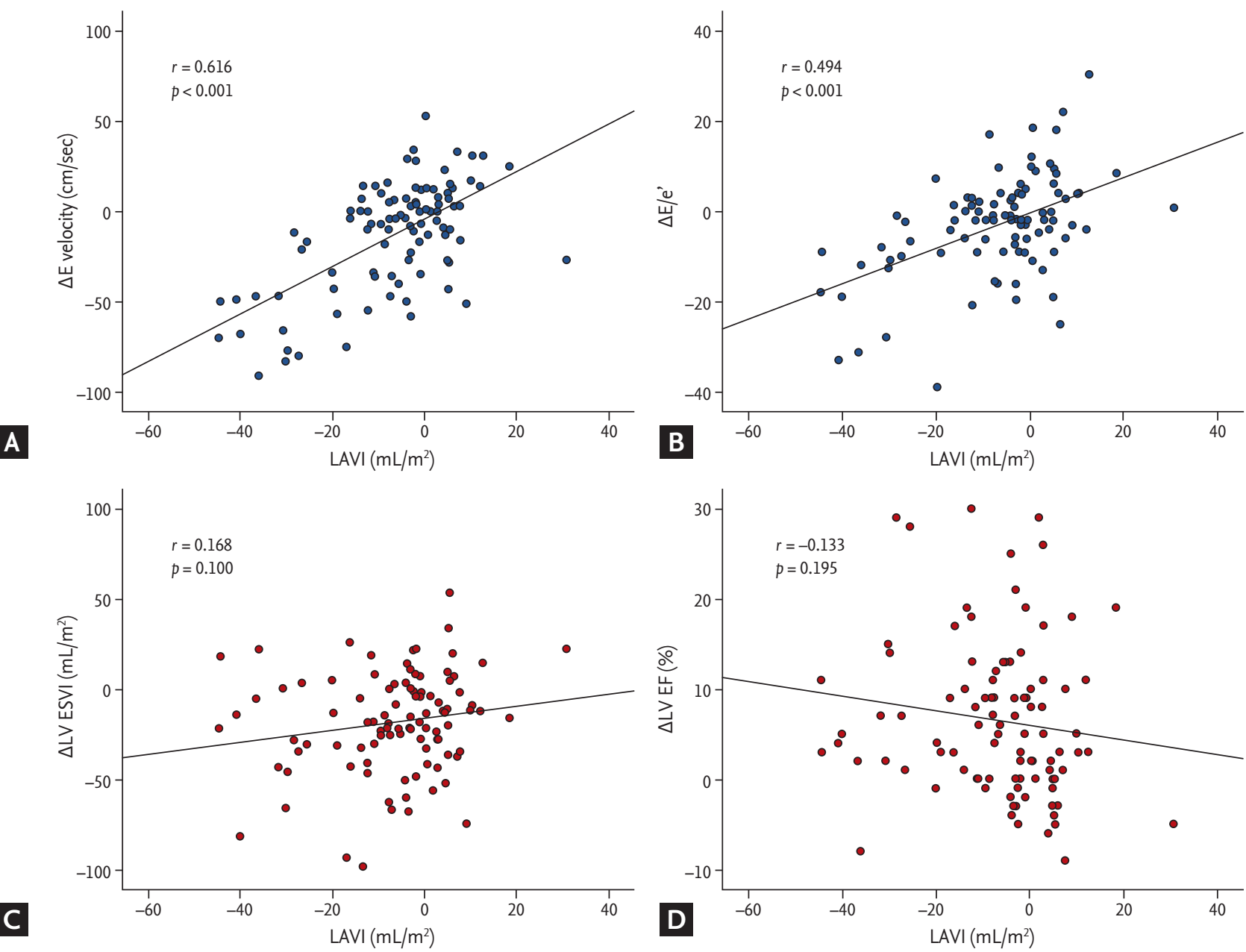

Figure 2. Simple correlation between changes in left atrial volume index (LAVI) and other echocardiographic variables. (A) Early mitral inflow velocity. (B) Early mitral to mitral annular velocity ratio. (C) Left ventricular end-systolic volume index (LV ESVI). (D) Left ventricular ejection fraction (LV EF). E velocity, early mitral inflow velocity; e' velocity, early mitral annular velocity.

$\left.\mathrm{mL} / \mathrm{m}^{2}, \mathrm{p}=0.006\right)$, as well as percent changes in LV ESVI $(-24.8 \% \pm 25.2 \%$ vs. $-12.7 \% \pm 26.4 \%)$. Table 4 demonstrates multivariate analysis for determinants of late LV response. LAVR at early FU (OR, 6.94; 95\% CI, 1.40 to $34.37 ; p=0.018)$ and LVVR at early FU (OR, 12.35; $95 \%$ CI, 2.65 to 57.62) were independent predictors for the late response, even after adjustment for gender, LBBB, QRS duration, and baseline LV ESVI and LAVI.

\section{Clinical events and LAVR}

Over the course of the study period, 26 patients (26.8\%) experienced the composite endpoint. Specifically, 13 patients (13.4\%) experienced death from cardiac causes and 13 patients (13.4\%) experienced inpatient admissions for heart failure. Fig. 5 shows event-free survival rates for the composite endpoint stratified by LAVR at early FU. Patients with early LAVR demonstrated a trend of favorable event-free survival compared to those without early LAVR (log-rank $p=0.066)$.

\section{DISCUSSION}

The principal findings of the current study examining longitudinal changes in LV and LA volume after CRT were that: (1) LAVR occurred early (median 6 months) after CRT whereas LVVR was sustained through the long-term FU period (median 30 months); (2) LAVR was 
Table 3. Factors associated with higher left atrial volume reduction at early follow-up

\begin{tabular}{|c|c|c|c|c|}
\hline \multirow{2}{*}{ Variable } & \multicolumn{2}{|c|}{ Univariate } & \multicolumn{2}{|c|}{ Multivariate } \\
\hline & OR $(95 \% \mathrm{CI})$ & $p$ value & OR (95\% CI) & $p$ value \\
\hline \multicolumn{5}{|c|}{ Baseline echocardiography } \\
\hline LV ESVI & $1.00(0.98-1.01)$ & 0.613 & - & - \\
\hline LV EF & $1.02(0.96-1.09)$ & 0.572 & - & - \\
\hline LAVI & $0.99(0.97-1.00)$ & 0.985 & - & - \\
\hline E velocity & $1.00(0.98-1.01)$ & 0.652 & - & - \\
\hline $\mathrm{E} / \mathrm{e}^{\prime}$ & $0.99(0.96-1.03)$ & 0.708 & - & - \\
\hline MR grade & $0.93(0.68-1.28)$ & 0.853 & - & - \\
\hline \multicolumn{5}{|c|}{ Early follow-up echocardiography } \\
\hline$\Delta$ LV ESVI & $1.01(1.00-1.02)$ & 0.011 & $1.01(0.99-1.03)$ & 0.363 \\
\hline$\Delta \mathrm{LV} \mathrm{EF}$ & $0.95(0.90-1.00)$ & 0.043 & $0.95(0.89-1.02)$ & 0.163 \\
\hline$\Delta \mathrm{E}$ velocity & $1.04(1.02-1.06)$ & $<0.001$ & $1.04(1.02-1.07)$ & 0.002 \\
\hline$\Delta \mathrm{E} / \mathrm{e}^{\prime}$ & $1.06(1.02-1.11)$ & 0.006 & $1.00(0.94-1.05)$ & 0.846 \\
\hline$\Delta \mathrm{MR}$ grade & $1.42(0.99-2.04)$ & 0.055 & $1.22(0.79-1.89)$ & 0.371 \\
\hline
\end{tabular}

OR, odds ratio; CI, confidence interval; LV, left ventricular; ESVI, end-systolic volume index; EF, ejection fraction; LAVI, left atrial volume index; E velocity, early mitral inflow velocity; e' velocity, early mitral annular velocity; MR, mitral regurgitation; $\Delta$ values, changes between baseline and early follow-up.
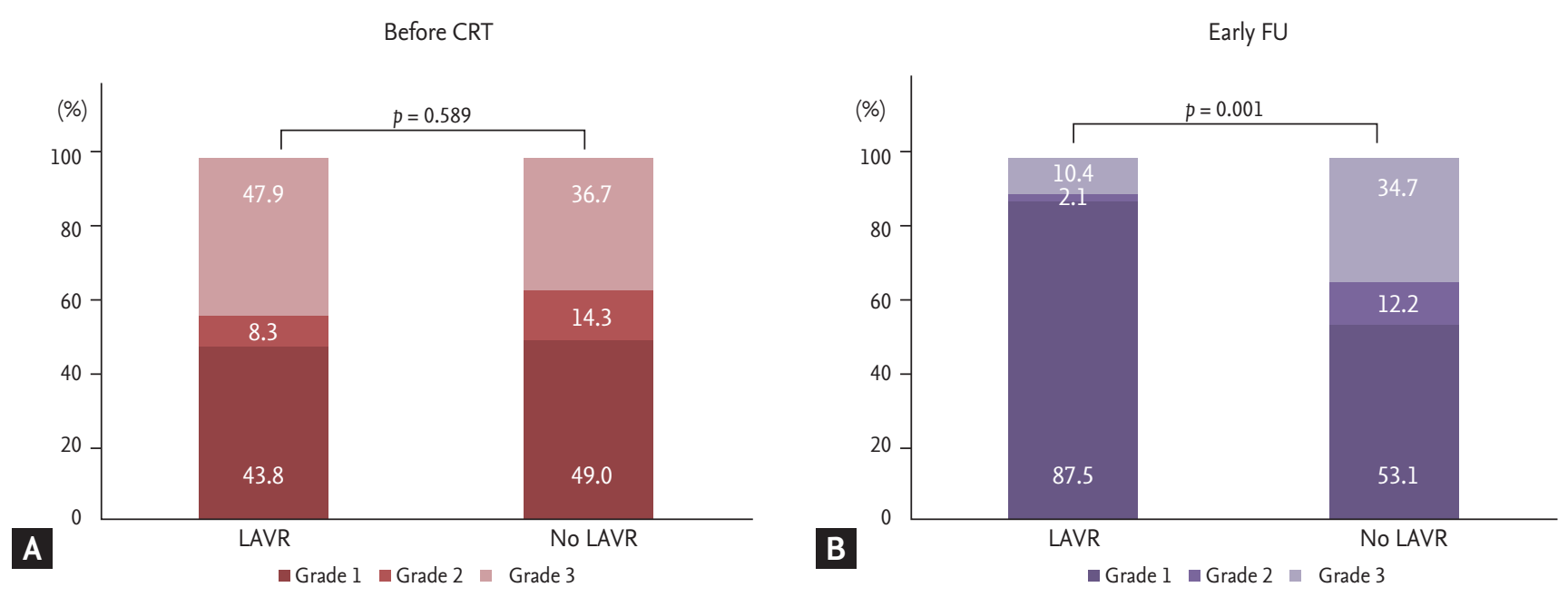

Figure 3. Comparison of changes in diastolic function before cardiac resynchronization therapy (CRT) and at early follow-up (FU) between left atrial volume reduction (LAVR) and no LAVR. (A) Diastolic function grade before CRT. (B) Diastolic function grade at early FU.

closely associated with changes in E velocity and mitral inflow pattern, suggesting its relation with LV filling pressure and optimization of mitral inflow pattern; (3) early significant LAVR was closely related to long-term LV response to CRT.

\section{Time course changes of LAVR and LVVR}

CRT induces favorable changes in LV activation pattern, thus improving cardiac performance and reducing LV ESV [12,13]. Because CRT response is usually defined according to the percentage of LV ESV reduction [14], LVVR is an important determining factor for outcomes and functional improvement in patients with CRT de- 

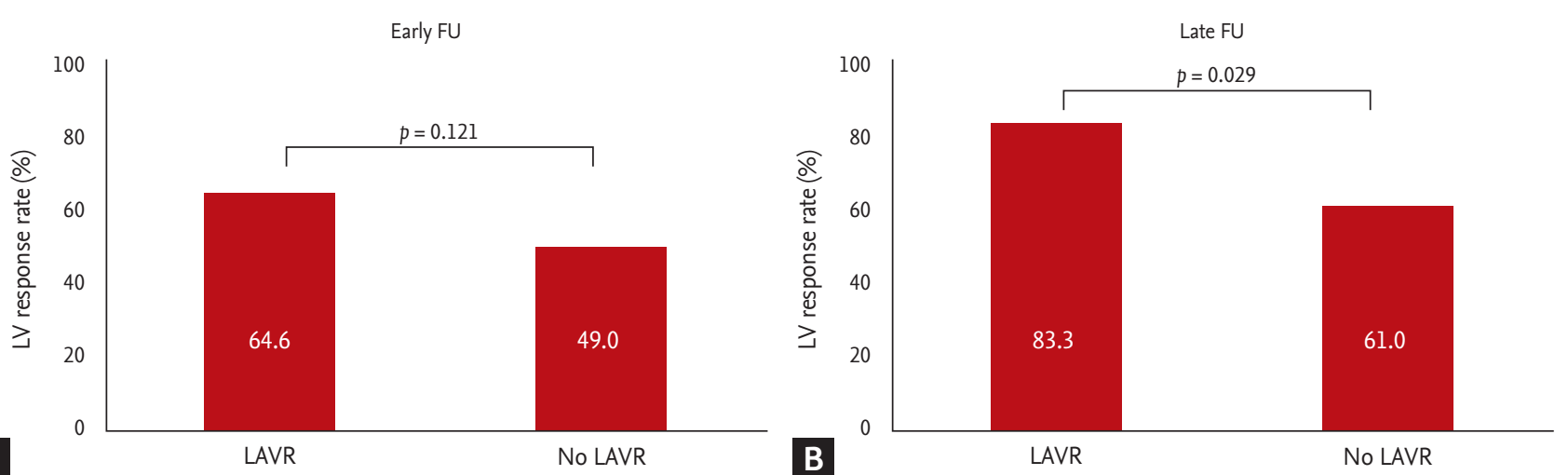

Figure 4. Comparison of left ventricular (LV) response between left atrial volume reduction (LAVR) and no LAVR. (A) Prevalence of LV response at early follow-up (FU). (B) Prevalence of LV response at late FU.

Table 4. Multivariate analysis for predictors of left ventricular response at late follow-up

\begin{tabular}{lccc}
\hline Variable & Odds ratio & 95\% Confidential interval & $p$ value \\
\hline Female gender & 1.61 & $0.35-7.43$ & 0.541 \\
LBBB & 3.55 & $0.67-18.84$ & 0.137 \\
QRS duration & 0.95 & $0.95-1.02$ & 0.292 \\
LV ESVI, baseline & 0.99 & $0.97-1.01$ & 0.150 \\
LAVI, baseline & 0.98 & $0.95-1.02$ & 0.342 \\
LAVR at early FU & 6.94 & $1.40-34.37$ & 0.018 \\
LVVR at early FU & 12.35 & $2.65-57.62$ & 0.001 \\
\hline
\end{tabular}

LBBB, left bundle branch block; LV, left ventricular; ESVI, end-systolic volume index; LAVI, left atrial volume index; LAVR, left atrial volume reduction; FU, follow-up; LVVR, left ventricular volume response.

vice implants [2]. LVVR after CRT was reported to be a better predictor of long-term survival than clinical response evaluated by improvement in New York Heart Association class or the 6 minutes walking test [15].

LVVR usually accompanies LAVR [16-18], but discrepant LAVR and LVVR has been reported in approximately $20 \%$ of patients at 1 year after CRT, in which patients with discordant LA and LVVR showed more favorable outcomes compared to lesser volume reduction of both chambers [5]. LAVR was maximized in the earlier period compared to LVVR and then stagnated, suggesting a discrepancy in the time-course changes of LA and LV volume that resulted in discrepant LAVR and LVVR at 1 year after CRT. LAVR was also a predictor for late LV response. Therefore, more favorable outcomes in patients with discordant LA and LVVR compared to lesser volume reduction of both chambers could be explained by this time gap in which LVVR follows LAVR. More than
1 year long-term FU is necessary to define LV response to CRT, especially in patients with a favorable LAVR during the early FU period.

\section{Associated factors with LAVR}

We found that $\Delta \mathrm{E}$ velocity or $\Delta \mathrm{E} / \mathrm{e}^{\prime}$ was more associated with early LAVR than $\Delta \mathrm{LV}$ ESV or $\Delta \mathrm{LV}$ EF. That is, LAVR was more closely related to hemodynamic variables during diastole, such as E velocity, E/e', and MR severity rather than LV volumetric or functional parameters. This finding also explains the discrepant LAVR and LVVR values after CRT [5], which might be because different factors determine LAVR and LVVR.

Interestingly, E velocity was the most important factor for early LAVR in our study. It was more important than $\mathrm{E} / \mathrm{e}^{\prime}$, a well-known estimation of LV filling pressure. E velocity is directly influenced by LA pressure and LV diastolic function, whereas $\mathrm{e}^{\prime}$ is influenced by LV relax- 


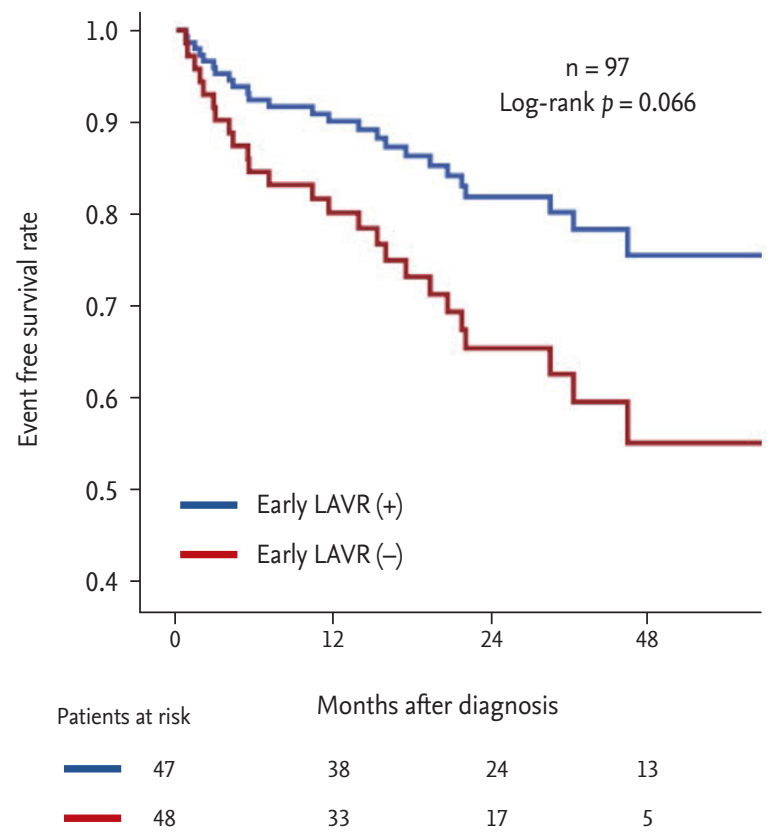

Figure 5. Comparison of event free survival rate for composite cardiovascular events stratified by left atrial volume reduction (LAVR) at early follow-up.

ation and independent of LA pressure. E/e', correcting E velocity for the influence of relaxation $\left(e^{\prime}\right)$, is traditionally considered to reflect LV filling pressure [19]. A plausible explanation for the stronger association of $\mathrm{E}$ velocity to LAVR over $\mathrm{E} / \mathrm{e}^{\prime}$ in the current study is that $\mathrm{e}^{\prime}$ is unreliable in LBBB or biventricular pacing, resulting in inaccurate $\mathrm{E} / \mathrm{e}^{\prime}$ for assessing LV filling pressure [20]. $E$ velocity adjusted by CRT optimization $[21,22]$ could be the most important determinant for LAVR following CRT since $\mathrm{E} / \mathrm{e}^{\prime}$ or $\mathrm{e}^{\prime}$ may be inaccurate in populations of electrical dyssynchrony and severe heart failure [22]. Larger in-depth studies are warranted to definitively identify any association between E velocity, diastolic functional recovery, and CRT optimization.

\section{LAVR as surrogate marker for CRT response}

Both LAVR and LVVR at early FU were independent factors for long-term CRT response. Since echocardiographic CRT response is usually defined using the criteria of $15 \%$ reduction of LVESV, it is not surprising that early LVVR was an independent predictor for long-term CRT response in the current study. More interestingly, early LAVR independently predicted future CRT response. LAVR has been reported to predict clinical outcomes in patients with a CRT device implant $[3,4,23]$. In addition to LV systolic function, several factors may be involved in the pathophysiology of LAVR in patients with heart failure after CRT device implantation. According to Frank-Starling's law, LA enlargement may occur as a compensatory mechanism to maintain LA stroke volume when LV filling pressure is elevated $[24,25]$. Reduction of LV filling pressure and LA pressure leads to decreased LA size. MR, caused by distortion of the mitral apparatus in the presence of dyssynchrony, may be reduced by CRT with a consequent decrease in LA pressure and volume [13]. CRT may induce LAVR through optimization of atrioventricular and interventricular delay [26-28], as efficient ventricle preloading through a properly timed atrial contraction has been shown to improve stroke work [29]. Considering the above-described factors, LAVR is a useful surrogate marker reflecting complex variables such as LA pressure, MR severity, and atrioventricular optimization after CRT device implantation. LAVR could be used as a surrogate marker for optimally adjusted status of CRT for each individual after device implantation. This explains why LAVR at early FU, a marker for early CRT optimization, predicted favorable LVVR during long-term FU. The clinical impact of early LAVR demonstrated a trend for favorable clinical outcomes (combined cardiac death and inpatient hospitalization) in the early LAVR group. Further studies with large numbers of patients are warranted.

\section{Limitations}

The current findings were based on a retrospective analysis, which prevents inference of a causal relationship among the variables. Nevertheless, all medical records and echocardiographic images were carefully reviewed to avoid possible biases. This was a pilot study, and further larger studies are required to conclude this hypothesis. Our study population consisted of a high prevalence of women and non-ischemic cardiomyopathy. Therefore, application of this data to other populations should be cautious. Data regarding functional status of the left atrium is lacking, and further studies using LA functional variables such as strain would be useful to better define the relationship of LA size and function to CRT response. Since changes in E velocity were related to changes in LV volume, early LA volume changes may 
be partially influenced by early changes in LV volume. However, mitral inflow pattern reflecting LV diastolic properties was also profoundly improved in the early LAVR group, suggesting improved diastolic dysfunction in the early phase of this group. Clinical data reflecting exercise performance, such as cardiopulmonary exercise tests and 6-minute walking tests, are lacking in this study. CRT optimization and medications, including dosages of each drug for heart failure, were administered according to the attending physician's preference for each patient. There was no standardized protocol for the entire study population.

\section{KEY MESSAGE}

1. Left atrial volume reduction (LAVR) significantly occurred within 1 year after cardiac resynchronization therapy (CRT).

2. LAVR at early follow-up (LAVR $\geq 7.5 \%$, within 1 year) was closed related to changes in early mitral inflow velocity.

3. LAVR at early follow-up was an independent predictor for the long-term left ventricular response to CRT.

\section{Conflict of interest}

No potential conflict of interest relevant to this article was reported.

\section{REFERENCES}

1. Yancy CW, Jessup M, Bozkurt B, et al. 2013 ACCF/AHA guideline for the management of heart failure: a report of the American College of Cardiology Foundation/American Heart Association Task Force on Practice Guidelines. J Am Coll Cardiol 2013;62:e147-e239.

2. Solomon SD, Foster E, Bourgoun M, et al. Effect of cardiac resynchronization therapy on reverse remodeling and relation to outcome: multicenter automatic defibrillator implantation trial: cardiac resynchronization therapy. Circulation 2010;122:985-992.

3. Kuperstein R, Goldenberg I, Moss AJ, et al. Left atrial volume and the benefit of cardiac resynchronization therapy in the MADIT-CRT trial. Circ Heart Fail 2014;7:154-16o.
4. Brenyo A, Link MS, Barsheshet A, et al. Cardiac resynchronization therapy reduces left atrial volume and the risk of atrial tachyarrhythmias in MADIT-CRT (Multicenter Automatic Defibrillator Implantation Trial with Cardiac Resynchronization Therapy). J Am Coll Cardiol 2011;58:1682-1689.

5. Mathias A, Moss AJ, McNitt S, et al. Clinical implications of complete left-sided reverse remodeling with cardiac resynchronization therapy: a MADIT-CRT Substudy. J Am Coll Cardiol 2016;68:1268-1276.

6. Ghio S, Freemantle N, Scelsi L, et al. Long-term left ventricular reverse remodelling with cardiac resynchronization therapy: results from the CARE-HF trial. Eur J Heart Fail 2009;11:480-488.

7. Tsang TS, Barnes ME, Gersh BJ, Bailey KR, Seward JB. Left atrial volume as a morphophysiologic expression of left ventricular diastolic dysfunction and relation to cardiovascular risk burden. Am J Cardiol 2002;90:1284-1289.

8. Lang RM, Bierig M, Devereux RB, et al. Recommendations for chamber quantification: a report from the American Society of Echocardiography's Guidelines and Standards Committee and the Chamber Quantification Writing Group, developed in conjunction with the European Association of Echocardiography, a branch of the European Society of Cardiology. J Am Soc Echocardiogr 2005;18:1440-1463.

9. Nagueh SF, Appleton CP, Gillebert TC, et al. Recommendations for the evaluation of left ventricular diastolic function by echocardiography. J Am Soc Echocardiogr 2009;22:107-133.

10. Rudski LG, Lai WW, Afilalo J, et al. Guidelines for the echocardiographic assessment of the right heart in adults: a report from the American Society of Echocardiography endorsed by the European Association of Echocardiography, a registered branch of the European Society of Cardiology, and the Canadian Society of Echocardiography. J Am Soc Echocardiogr 2010;23:685-713.

11. Zoghbi WA, Adams D, Bonow RO, et al. Recommendations for noninvasive evaluation of native valvular regurgitation: a report from the American Society of Echocardiography developed in collaboration with the Society for Cardiovascular Magnetic Resonance. J Am Soc Echocardiogr 2017;30:303-371.

12. Bax JJ, Abraham T, Barold SS, et al. Cardiac resynchronization therapy: Part 2. Issues during and after device implantation and unresolved questions. J Am Coll Cardiol 
2005;46:2168-2182.

13. Yu CM, Chau E, Sanderson JE, et al. Tissue Doppler echocardiographic evidence of reverse remodeling and improved synchronicity by simultaneously delaying regional contraction after biventricular pacing therapy in heart failure. Circulation 2002;105:438-445.

14. Mele D, Bertini M, Malagu M, Nardozza M, Ferrari R. Current role of echocardiography in cardiac resynchronization therapy. Heart Fail Rev 2017;22:699-722.

15. Bertini M, Hoke U, van Bommel RJ, et al. Impact of clinical and echocardiographic response to cardiac resynchronization therapy on long-term survival. Eur Heart J Cardiovasc Imaging 2013;14:774-781.

16. Donal E, Tan K, Leclercq C, et al. Left atrial reverse remodeling and cardiac resynchronization therapy for chronic heart failure patients in sinus rhythm. J Am Soc Echocardiogr 2009;22:1152-1158.

17. Yu CM, Fang F, Zhang Q, et al. Improvement of atrial function and atrial reverse remodeling after cardiac resynchronization therapy for heart failure. J Am Coll Cardiol 2007;50:778-785.

18. D'Andrea A, Caso P, Romano S, et al. Different effects of cardiac resynchronization therapy on left atrial function in patients with either idiopathic or ischaemic dilated cardiomyopathy: a two-dimensional speckle strain study. Eur Heart J 2007;28:2738-2748.

19. Nagueh SF, Middleton KJ, Kopelen HA, Zoghbi WA, Quinones MA. Doppler tissue imaging: a noninvasive technique for evaluation of left ventricular relaxation and estimation of filling pressures. J Am Coll Cardiol 1997;30:1527-1533.

20. Marwick TH. The role of echocardiography in heart failure. J Nucl Med 2015;56 Suppl 4:31S-38S.

21. Brignole M, Auricchio A, Baron-Esquivias G, et al. 2013 ESC guidelines on cardiac pacing and cardiac resynchronization therapy: the task force on cardiac pacing and resynchronization therapy of the European Society of
Cardiology (ESC). Developed in collaboration with the European Heart Rhythm Association (EHRA). Eur Heart J 2013;34:2281-2329.

22. Mullens W, Borowski AG, Curtin RJ, Thomas JD, Tang WH. Tissue Doppler imaging in the estimation of intracardiac filling pressure in decompensated patients with advanced systolic heart failure. Circulation 2009;119:6270.

23. Kloosterman M, Rienstra M, Mulder BA, Van Gelder IC, Maass AH. Atrial reverse remodelling is associated with outcome of cardiac resynchronization therapy. Europace 2016;18:1211-1219.

24. Anwar AM, Geleijnse ML, Soliman OI, Nemes A, ten Cate FJ. Left atrial Frank-Starling law assessed by real-time, three-dimensional echocardiographic left atrial volume changes. Heart 2007;93:1393-1397.

25. Obokata M, Negishi K, Kurosawa K, et al. Incremental diagnostic value of la strain with leg lifts in heart failure with preserved ejection fraction. JACC Cardiovasc Imaging 2013;6:749-758.

26. Pan G, Liu Z, He P, et al. Effect of periodic pacemaker optimization on left atrial reverse remodeling in long-term cardiac resynchronization therapy. J Interv Card Electrophysiol 2014;39:87-93.

27. Mullens W, Grimm RA, Verga T, et al. Insights from a cardiac resynchronization optimization clinic as part of a heart failure disease management program. J Am Coll Cardiol 2009;53:765-773.

28. Mullens W, Kepa J, De Vusser P, et al. Importance of adjunctive heart failure optimization immediately after implantation to improve long-term outcomes with cardiac resynchronization therapy. Am J Cardiol 2011;108:409-415.

29. Hu Y, Gurev V, Constantino J, Trayanova N. Efficient preloading of the ventricles by a properly timed atrial contraction underlies stroke work improvement in the acute response to cardiac resynchronization therapy. Heart Rhythm 2013;10:1800-1806. 